\title{
CLOSED FORM FORMULA FOR THE NUMBER OF RESTRICTED COMPOSITIONS
}

\author{
GAŠPER JAKLIČ, VITO VITRIH ${ }^{凶}$ and EMIL ŽAGAR
}

(Received 17 June 2009)

\begin{abstract}
In this paper, compositions of a natural number are studied. The number of restricted compositions is given in a closed form, and some applications are presented.
\end{abstract}

2000 Mathematics subject classification: primary 05A17; secondary 68R05.

Keywords and phrases: composition, partition, restricted, maximal element, minimal element.

\section{Introduction}

Compositions and partitions of a natural number $n$ frequently appear in research and in practical applications. Although the number of compositions or partitions satisfying particular requirements can be obtained from their generating functions, this is a serious drawback, since it requires symbolic computational facilities and exact computations, because of the computational complexity involved. In this paper, we present a closed form formula for the number of restricted compositions, and give some applications of the results.

Let us be more precise. The list of natural numbers $t_{i}$, which sum to a natural number $n$, is an integer composition of $n$. The set of all such lists, where the ordering of the summands matters, is the set of all integer compositions of $n$. The set of restricted integer compositions of $n$ is the subset of all compositions that satisfy some additional restrictions, for example, on the number of summands, on the values of summands, and so on. Let $a, b, n \in \mathbb{N}$ with $a \leq b \leq n$. Let $C(n, a, b)$ denote the number of compositions of $n$, such that summands $t_{i}$ are natural numbers, bounded as $a \leq t_{i} \leq b$, for all $i$. Furthermore, let $C(n, k, a, b)$ denote the number of those restricted compositions of $n$, where the number of summands is equal to $k$,

$$
\sum_{i=1}^{k} t_{i}=n, \quad a \leq t_{j} \leq b, j=1,2, \ldots, k .
$$

(C) 2010 Australian Mathematical Publishing Association Inc. 0004-9727/2010 \$16.00 
Clearly,

$$
C(n, a, b)=\sum_{k=\lceil n / b\rceil}^{\lfloor n / a\rfloor} C(n, k, a, b) .
$$

It is trivial to prove that

$$
C(n):=C(n, 1, n)=2^{n-1} \quad \text { and } \quad C(n, k):=C(n, k, 1, n)=\left(\begin{array}{l}
n-1 \\
k-1
\end{array}\right) .
$$

There are also known formulas for the special cases

$$
C(n, k, a, n)=\left(\begin{array}{c}
n-k a+k-1 \\
k-1
\end{array}\right), \quad C(n, a, n)=\sum_{k=1}^{\lfloor n / a\rfloor} C(n, k, a, n) .
$$

Also an obvious recursive relation for the general case

$$
C(n, k, a, b)=\sum_{i=n-b}^{n-a} C(i, k-1, a, b)
$$

is right at hand. Nevertheless, generating functions are known for both $C(n, k, a, b)$ and $C(n, a, b)$. They are of the form $[2,6]$

$$
\left(z^{a} \frac{1-z^{b-a+1}}{1-z}\right)^{k} \text { and } \frac{1}{1-z^{a}\left(\left(1-z^{b-a+1}\right) /(1-z)\right)},
$$

respectively. We are also interested in a closed form formula for the number of compositions of $n$ with more than one maximal (or minimal) element. We will denote them by $\operatorname{Max}(n)$ and $\operatorname{Min}(n)$, respectively. Again, there are generating functions known for $C(n)-\operatorname{Max}(n)$ and $C(n)-\operatorname{Min}(n)$, which are of the form

$$
(1-z)^{2} \sum_{j=1}^{\infty}\left(\frac{z^{j}}{1-2 z+z^{j+1}}\right)^{2} \text { and }(1-z)^{2} \sum_{j=1}^{\infty}\left(\frac{z^{j}}{1-z-z^{j}}\right)^{2},
$$

respectively [6].

It is quite easy to obtain closed form formulas at least for $C(n, 1, b)$ and $C(n, a, b)$, $a>1$. Namely, by (1.1),

$$
\frac{1}{1-z\left(\left(1-z^{b}\right) /(1-z)\right)}=\sum_{n=0}^{\infty} C(n, 1, b) z^{n}
$$

Since

$$
\begin{aligned}
\frac{1}{1-z\left(\left(1-z^{b}\right) /(1-z)\right)} & =\frac{1-z}{1-2 z+z^{b+1}}=(1-z) \sum_{i=0}^{\infty}\left(2 z-z^{b+1}\right)^{i} \\
& =(1-z) \sum_{i=0}^{\infty} \sum_{j=0}^{i}(-1)^{j}\left(\begin{array}{l}
i \\
j
\end{array}\right) 2^{i-j} z^{i-j} z^{j(b+1)}
\end{aligned}
$$


the coefficient at $z^{n}$ becomes

$$
C(n, 1, b)=g(n, b)-g(n-1, b),
$$

where

$$
g(n, b):=\sum_{\substack{i, j \\
i+j b=n}}(-1)^{j}\left(\begin{array}{l}
i \\
j
\end{array}\right) 2^{i-j}
$$

Similarly $C(n, a, b)=g(n, a, b)-g(n-1, a, b), a>1$, where

$$
g(n, a, b)=\sum_{\substack{i, j, \ell \\
i+j(a-1)+\ell(b-a+1)=n}}(-1)^{\ell}\left(\begin{array}{l}
i \\
j
\end{array}\right)\left(\begin{array}{l}
j \\
\ell
\end{array}\right) .
$$

But it seems that deriving an explicit formula for $C(n, k, a, b)$ is a far more difficult problem.

The paper is organized as follows. In Section 2 closed form formulae for the number of restricted compositions and restricted partitions are obtained. These are used as a basis for studying two related problems in Section 3. The paper concludes with some examples in Section 4.

\section{Restricted compositions}

In this section, our aim is to find a combinatorial closed form expression for $C(n, k, a, b)$.

THEOREM 2.1. Let $a \leq b \leq n$ and $\lceil n / b\rceil \leq k \leq\lfloor n / a\rfloor$. To each composition of $n$ assign a vector $\boldsymbol{i}=\left(i_{2}, i_{3}, \ldots, i_{b}\right)$, where $i_{j}$ denotes the frequency of the number $j$ in the composition. Moreover, let

$$
\begin{aligned}
& \alpha_{j}:=n-k(j-1)-\sum_{\ell=j+1}^{b}(\ell-j+1) i_{\ell}, \quad \beta_{j}:=k-\sum_{\ell=j+1}^{b} i_{\ell}, \\
& \gamma_{j}:=\left\lfloor\frac{n-k-\sum_{\ell=j+1}^{b}(\ell-1) i_{\ell}}{j-1}\right\rfloor, \quad j=2,3, \ldots, b .
\end{aligned}
$$

Then:

(a) $C(n, k, 1, b)=\sum_{\substack{i_{2}=\alpha_{2}, i_{3}, \ldots, i_{b} \\ \max \left\{0, \alpha_{j}\right\} \leq i_{j} \leq \min \left\{\beta_{j}, \gamma_{j}\right\}}} \prod_{\ell=2}^{b}\left(\begin{array}{c}k-\sum_{j=2}^{\ell-1} i_{j} \\ i_{\ell}\end{array}\right)$;

(b) $C(n, k, a, b)=C(n-k(a-1), k, 1, b-(a-1))$;

(c) if $(k b-n) /(k-1) \in \mathbb{N}$ and $(k a+(b-a)-n) /(k-1) \in \mathbb{N}_{0}$, then

$$
C(n, k, a, b)=\left(\begin{array}{c}
n-k a+k-1 \\
k-1
\end{array}\right) .
$$


PROOF. First, note that the frequency of number 1 is $n-\sum_{\ell=2}^{b} \ell i_{\ell}$ and so the number of summands in the composition is

$$
k(\boldsymbol{i}):=n-\sum_{\ell=2}^{b}(\ell-1) i_{\ell} .
$$

Furthermore, there are exactly

$$
\prod_{\ell=2}^{b}\left(\begin{array}{c}
k(\boldsymbol{i})-\sum_{j=2}^{\ell-1} i_{j} \\
i_{\ell}
\end{array}\right)
$$

different compositions with the same vector $\boldsymbol{i}$. Since the number of summands has to be $k$, the only admissible compositions are those with $k(\boldsymbol{i})=k$. Therefore, the relations

$$
\left(k-\sum_{\ell=j}^{b} i_{\ell}\right)(j-1) \geq n-\sum_{\ell=j}^{b} \ell i_{\ell} \geq k-\sum_{\ell=j}^{b} i_{\ell} \geq 0, \quad j=2,3, \ldots, b,
$$

have to be satisfied. With the help of (2.1), we obtain the appropriate ranges for numbers $i_{j}$,

$$
\max \left\{0, \alpha_{j}\right\} \leq i_{j} \leq \min \left\{\beta_{j}, \gamma_{j}\right\}, \quad 3 \leq j \leq b, i_{2}:=\alpha_{2}=\gamma_{2} .
$$

The first formula is therefore proven. In order to show that an additional condition, which requires the summands in the composition to be at least $a$, does not increase the difficulty of the problem, let us define a function

$$
\begin{aligned}
& f:\left\{\left(t_{1}, \ldots, t_{k}\right), \sum_{i=1}^{k} t_{i}=n, a \leq t_{i} \leq b\right\} \\
& \quad \rightarrow\left\{\left(s_{1}, \ldots, s_{k}\right), \sum_{i=1}^{k} s_{i}=n-k(a-1), 1 \leq s_{i} \leq b-(a-1)\right\}, \\
& f:\left(t_{1}, t_{2}, \ldots, t_{k}\right) \mapsto\left(t_{1}-(a-1), t_{2}-(a-1), \ldots, t_{k}-(a-1)\right),
\end{aligned}
$$

which is clearly a bijection and thus

$$
C(n, k, a, b)=C(n-k(a-1), k, 1, b-(a-1)) .
$$

To prove the last statement of the theorem, assume $C(n, k, a, b)=C\left(m, k, a_{2}, m\right)$. Then $m=n+k(m-b)$ and $a_{2}=a+m-b$. Hence

$$
m=\frac{k b-n}{k-1}, \quad a_{2}=\frac{k a+(b-a)-n}{k-1} .
$$

If $m \in \mathbb{N}$ and $a_{2} \in \mathbb{N}_{0}$, then $C\left(m, k, a_{2}, m\right)$ is well defined and it follows that

$$
\begin{aligned}
C(n, k, a, b) & =C\left(m, k, a_{2}, m\right)=C\left(m-k a_{2}, k, 0, m-k a_{2}\right) \\
& =\left(\begin{array}{c}
n-k a+k-1 \\
k-1
\end{array}\right) .
\end{aligned}
$$


This result can be used to derive some interesting properties of restricted compositions.

COROLLARY 2.2. The following formulae hold true:

$$
\begin{aligned}
& C(n, k, 1,2)=\left(\begin{array}{c}
k \\
n-k
\end{array}\right), \quad C(n, 1,2)=\sum_{k=\lceil n / 2\rceil}^{n}\left(\begin{array}{c}
k \\
n-k
\end{array}\right)=\sum_{k=0}^{\lfloor n / 2\rfloor}\left(\begin{array}{c}
n-k \\
k
\end{array}\right), \\
& C(n, k, a, a+1)=\left(\begin{array}{c}
k \\
n-k a
\end{array}\right), \quad C(n, a, a+1)=\sum_{k=\lceil n / a+1\rceil}^{\lfloor n / a\rfloor}\left(\begin{array}{c}
k \\
n-k a
\end{array}\right) .
\end{aligned}
$$

Suppose now that one is interested in restricted partitions. The list of natural numbers, which sum to $n$ and where the ordering of summands is not important, is the set of integer partitions of $n$. The partitions, where the number of summands is equal to $k$ and where they are bounded between $a$ and $b$, will be denoted by $P(n, k, a, b)$. The following corollary follows directly from Theorem 2.1.

COROLlary 2.3. Let $1 \leq a \leq b \leq n$ and $\lceil n / b\rceil \leq k \leq\lfloor n / a\rfloor$. To each partition of $n$ assign a vector $\boldsymbol{i}=\left(i_{2}, i_{3}, \ldots, i_{b}\right)$, where $i_{j}$ denotes the frequency of the number $j$ in the partition. Moreover, let $\alpha_{j}, \beta_{j}$ and $\gamma_{j}, j=2,3, \ldots, b$, be as in Theorem 2.1. Then:

(a) $P(n, k, 1, b)=\sum_{\substack{i_{2}=\alpha_{2}, i_{3}, \ldots, i_{b} \\ \max \left\{0, \alpha_{j}\right\} \leq i_{j} \leq \min \left\{\beta_{j}, \gamma_{j}\right\}}} 1$;

(b) $P(n, k, a, b)=P(n-k(a-1), k, 1, b-(a-1))$.

\section{Two related problems}

It is interesting to consider the problem of counting the compositions where more than one maximal (or minimal) summand exists. An application will be given in the final section. Using Theorem 2.1, one can prove the following theorem.

THEOREM 3.1. Let $\operatorname{Max}(n)$ denote the number of all compositions of $n$, such that there are at least two maximal summands, and let $\operatorname{Min}(n)$ denote the number of all compositions of $n$, such that there are at least two minimal summands. Then

$$
\begin{gathered}
\operatorname{Max}(n)=1+\sum_{i=2}^{\lfloor n / 2\rfloor} \sum_{v_{i}=2}^{\lfloor n / i\rfloor} \sum_{k=\left\lceil\left(n-i v_{i}\right) /(i-1)\right\rceil}^{n-i v_{i}}\left(\begin{array}{c}
k+v_{i} \\
v_{i}
\end{array}\right) C\left(n-i v_{i}, k, 1, i-1\right), \\
\operatorname{Min}(n)=\sum_{i=1}^{\lfloor n / 2\rfloor} \sum_{v_{i}=2}^{\lfloor n / i\rfloor} \sum_{k=\operatorname{sign}\left(n-i v_{i}\right)}^{\left\lfloor\left(n-i v_{i}\right) /(i+1)\right\rfloor}\left(\begin{array}{c}
k+v_{i} \\
v_{i}
\end{array}\right) C\left(n-i v_{i}, k, i+1, n-i v_{i}\right) .
\end{gathered}
$$

PROOF. Let us denote the value of maximal summands by $i$ and the frequency of $i$ in the composition by $v_{i}$. If $i=1$, then there is exactly one appropriate composition. Now let $i \in\{2,3, \ldots,\lfloor n / 2\rfloor\}$ and $v_{i} \in\{2,3, \ldots,\lfloor n / i\rfloor\}$. Consider the summands 
TABLE 1. Values of $\operatorname{Max}(n)$ and $\operatorname{Min}(n)$ for $n \leq 13$.

\begin{tabular}{cccccccccccccc}
\hline$n$ & 1 & 2 & 3 & 4 & 5 & 6 & 7 & 8 & 9 & 10 & 11 & 12 & 13 \\
\hline $\operatorname{Max}(n)$ & 0 & 0 & 0 & 1 & 3 & 8 & 17 & 36 & 72 & 144 & 286 & 569 & 1133 \\
$\operatorname{Min}(n)$ & 0 & 1 & 1 & 5 & 8 & 21 & 44 & 94 & 197 & 416 & 857 & 1766 & 3621 \\
\hline
\end{tabular}

which are smaller than $i$, and denote the number of these summands by $k:=k\left(i, v_{i}\right)$. Clearly $\left\lceil\left(n-i v_{i}\right) /(i-1)\right\rceil \leq k \leq n-i v_{i}$. Then there are $C\left(n-i v_{i}, k, 1, i-1\right)$ different possible compositions among them. But now the maximal summands could be arranged through the sequence of summands, which implies $\left(\begin{array}{c}k+v_{i} \\ v_{i}\end{array}\right)$ possibilities of where to set these $v_{i}$ maximal summands.

To prove the second formula, let $i$ denote the value of minimal summands. Therefore $i \in\{1,2, \ldots,\lfloor n / 2\rfloor\}$, and $v_{i} \in\{2,3, \ldots,\lfloor n / i\rfloor\}$. Now let $k$ denote the number of summands which are greater than $i$. If $n-i v_{i}=0$, then $k=0$ and there is exactly one such composition. Suppose that $n-i v_{i}>0$. If $\left\lfloor\left(n-i v_{i}\right) /(i+1)\right\rfloor=0$, there is no appropriate composition containing $v_{i}$ summands $i$, otherwise $k$ can be any number between 1 and $\left\lfloor\left(n-i v_{i}\right) /(i+1)\right\rfloor$. Further, there are exactly

$$
\left(\begin{array}{c}
k+v_{i} \\
v_{i}
\end{array}\right) C\left(n-i v_{i}, k, i+1, n-i v_{i}\right)
$$

compositions containing $v_{i}$ summands $i$ and $k$ summands greater than $i$.

Let $\operatorname{Max}_{C}(n)\left(\operatorname{Min}_{C}(n)\right)$ denote the number of compositions of $n$, such that there is exactly one maximal (minimal) summand, respectively. Since $\operatorname{Max}(n)+$ $\operatorname{Max}_{C}(n)=C(n)=2^{n-1}$ and $\operatorname{Min}(n)+\operatorname{Min}_{C}(n)=C(n), \operatorname{Max}(n)$ and $\operatorname{Min}(n)$ can also be computed via $\operatorname{Max}_{C}(n)$ and $\operatorname{Min}_{C}(n)$.

Corollary 3.2. Let $\operatorname{Max}(n)$ and $\operatorname{Min}(n)$ be as in Theorem 3.1. Then

$$
\begin{gathered}
\operatorname{Max}_{\mathrm{C}}(n)=\sum_{i=2}^{n} \sum_{k=\lceil(n-i) /(i-1)\rceil}^{n-i}(k+1) C(n-i, k, 1, i-1), \\
\operatorname{Min}_{C}(n)=\sum_{i=1}^{n} \sum_{k=\operatorname{sign}(n-i)}^{\lfloor(n-i) /(i+1)\rfloor}(k+1) C(n-i, k, i+1, n-i) .
\end{gathered}
$$

PROOF. The expressions can be obtained similarly as in the proof of Theorem 3.1.

Although it seems easier to obtain $\operatorname{Max}(n)$ and $\operatorname{Min}(n)$ from $\operatorname{Max}_{C}(n)$ and $\operatorname{Min}_{C}(n)$, we observe that the time complexity increases this way.

The next important question is the asymptotic behavior of $\operatorname{Max}(n)$ and $\operatorname{Min}(n)$ for large integers $n$. Numerical examples and Table 1 point to the following conjecture.

Conjecture 3.3. Let $\operatorname{Max}(n)$ and $\operatorname{Min}(n)$ be as in Theorem 3.1. Then

$$
\lim _{n \rightarrow \infty} \frac{\operatorname{Max}(n+1)}{\operatorname{Max}(n)}=\lim _{n \rightarrow \infty} \frac{\operatorname{Min}(n+1)}{\operatorname{Min}(n)}=2 .
$$




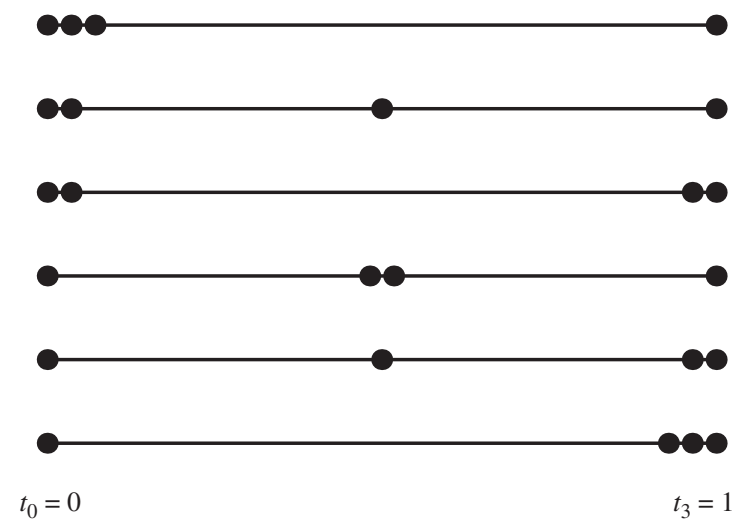

FIGURE 1. All possible cases for $n=3$.

\section{Examples}

An interesting application of $\operatorname{Max}(n)$ arises in numerical analysis, in particular in asymptotic analysis of the geometric Lagrange interpolation problem by Pythagoreanhodograph $(\mathrm{PH})$ curves $[1,5]$. Here the number of cases of the problem considered that need to be studied can be significantly reduced by knowing $\operatorname{Max}(n)$ in advance. More precisely, if the geometric interpolation (see [3], for example) by PH curves of degree $n$ is considered, the unknown interpolating parameters $t_{i}, i=1,2, \ldots, n-1$, have to lie in

$$
\mathcal{D}=\left\{\left(t_{i}\right)_{i=1}^{n-1} \in \mathbb{R}^{n-1} \mid t_{0}:=0<t_{1}<t_{2}<\cdots<t_{n-1}<1=: t_{n}\right\} .
$$

It turns out that the interpolation problem requires the analysis of a particular nonlinear system of equations involving the unknown $t_{i}$ only at the boundary of $\mathcal{D}$. Quite clearly, if the point in $\mathbb{R}^{n-1}$ is to be on the boundary of $\mathcal{D}$, at least two consecutive $t_{i}$ have to coincide (but not all of them, since $t_{0}=0$ and $t_{n}=1$ ). Thus the number of cases considered is equal to $C(n+1)-2=2^{n}-2$ (see Figure 1, for example).

Some further observations reduce the problem to the analysis of particular parts of the boundary. Let

$$
v_{i}:= \begin{cases}0 & t_{i-1} \neq t_{i}, \\ \max _{0 \leq j \leq i-1}\left\{i-j \mid t_{\ell+1}=t_{\ell}, j \leq \ell \leq i-1\right\} & \text { otherwise, }\end{cases}
$$

where $i=1,2, \ldots, n$. It turns out that if the sequence $\left(v_{i}\right)_{i=1}^{n}$ has a unique maximum, the corresponding choice of parameters $\left(t_{i}\right)_{i=1}^{n-1}$ can be skipped in the analysis. But the number of sequences $\left(v_{i}\right)_{i=1}^{n}$ for which the maximum is not unique is precisely $\operatorname{Max}(n+1)$.

Let us conclude the paper with an another example. In high order parametric polynomial approximation of circular arcs ([4], for example), the coefficients 
of the optimal solution involve the number of restricted partitions of a natural number. Namely, the coefficients of the parametric polynomial approximant $\boldsymbol{p}(t)=$ $(x(t), y(t))^{T}$, where

$$
x(t):=\sum_{k=0}^{n} \alpha_{k} t^{k}, \quad y(t):=\sum_{k=0}^{n} \beta_{k} t^{k},
$$

are of the form

$$
\alpha_{k}= \begin{cases}\sum_{j=0}^{k(n-k)} \widetilde{P}(j, k, n-k) \cos \left(\frac{k^{2} \pi}{2 n}+\frac{\pi}{n} j\right) & k \text { is even } \\ 0 & k \text { is odd }\end{cases}
$$

and

$$
\beta_{k}= \begin{cases}0 & k \text { is even, } \\ \sum_{j=0}^{k(n-k)} \widetilde{P}(j, k, n-k) \sin \left(\frac{k^{2} \pi}{2 n}+\frac{\pi}{n} j\right) & k \text { is odd },\end{cases}
$$

where $\widetilde{P}(n, k, b):=\sum_{\ell=1}^{k} P(n, \ell, 1, b)$.

\section{Acknowledgements}

We would like to thank Professor Marko Petkovšek and Professor Herbert Wilf for their valuable suggestions.

\section{References}

[1] R. T. Farouki, Pythagorean-Hodograph Curves: Algebra and Geometry Inseparable, Geometry and Computing, 1 (Springer, Berlin, 2008).

[2] P. Flajolet and R. Sedgewick, Analytic Combinatorics (Cambridge University Press, Cambridge, 2009).

[3] G. Jaklič, J. Kozak, M. Krajnc and E. Žagar, 'On geometric interpolation by planar parametric polynomial curves', Math. Comp. 76(260) (2007), 1981-1993.

[4] G. Jaklič, J. Kozak, M. Krajnc and E. Žagar, 'On geometric interpolation of circle-like curves', Comput. Aided Geom. Design 24(5) (2007), 241-251.

[5] G. Jaklič, J. Kozak, M. Krajnc, V. Vitrih and E. Žagar, 'Geometric Lagrange interpolation by planar cubic Pythagorean-hodograph curves', Comput. Aided Geom. Design 25(9) (2008), 720-728.

[6] N. J. A. Sloan, The on-line encyclopedia of integer sequences (2008), http://www.research.att.com/ njas/sequences.

GAŠPER JAKLIČ, FMF and IMFM, University of Ljubljana, Jadranska 19, Ljubljana, Slovenia

and

PINT, University of Primorska, Muzejski trg 2, Koper, Slovenia

e-mail: gasper.jaklic@fmf.uni-lj.si 
VITO VITRIH, PINT, University of Primorska, Muzejski trg 2, Koper, Slovenia e-mail: vito.vitrih@upr.si

EMIL ŽAGAR, FMF and IMFM, University of Ljubljana, Jadranska 19, Ljubljana, Slovenia

e-mail: emil.zagar@fmf.uni-lj.si 